\title{
Intravascular stenting of systemic venous baffle stenosis after corrective surgery for double outlet right ventricle with left isomerism
}

Takuya Miura, Tetsuya Sano, Hikaru Matsuda

\begin{abstract}
A 14 year old girl with double outlet right ventricle, left isomerism, and complicated atrial anatomy had undergone corrective surgery with intra-atrial rerouting at 5 years of age. Cardiac catheterisation eight years after the surgery showed that she had two systemic venous baffle stenoses: between the hepatic vein and caval vein, and the caval vein and right atrium. Two Palmaz stents were successfully implanted percutaneously through a long sheath and a balloon catheter. The stenoses were relieved immediately and her symptoms quickly disappeared.

(Heart 1999;81:218-220)
\end{abstract}

Keywords: stents; congenital heart defects; baffle stenoses

The use of stents in paediatric patients has been widely accepted, application of stents for venous obstruction in these patients is limited. ${ }^{1-4}$ We report a case of successful stent placement for multiple stenoses of systemic venous baffles after complicated corrective surgery for double outlet right ventricle with left isomerism.

First Department of Surgery, Osaka University Medical

School, Suita, 2-2

Yamadaoka, Suita, Osaka, 565-0871, Japan

T Miura

H Matsuda

Department of Pediatrics, Osaka

University Medical

School

T Sano

Correspondence to: $\mathrm{Dr}$ Matsuda.

Accepted for publication 29 July 1998

\section{Case report}

This female patient was born with moderate cyanosis and was referred to our hospital for surgical treatment when she was 5 years old. Preoperative evaluation elucidated that she had double outlet right ventricle with many associated anomalies: subaortic ventricular septal defect, atrial septal defect, severe pulmonary stenosis, patent ductus arteriosus, no right superior vena cava (SVC), a left SVC, an interrupted inferior vena cava, and direct drainage of the hepatic vein into the left atrium. All systemic venous return from the lower body except the hepatic vein was connected to the left SVC through the hemiazygous vein. All pulmonary veins were connected to the same atrium (fig 1A). She also had congenital complete atrioventricular block. Chest radiography showed a widely spread main bronchus indicating left isomerism.
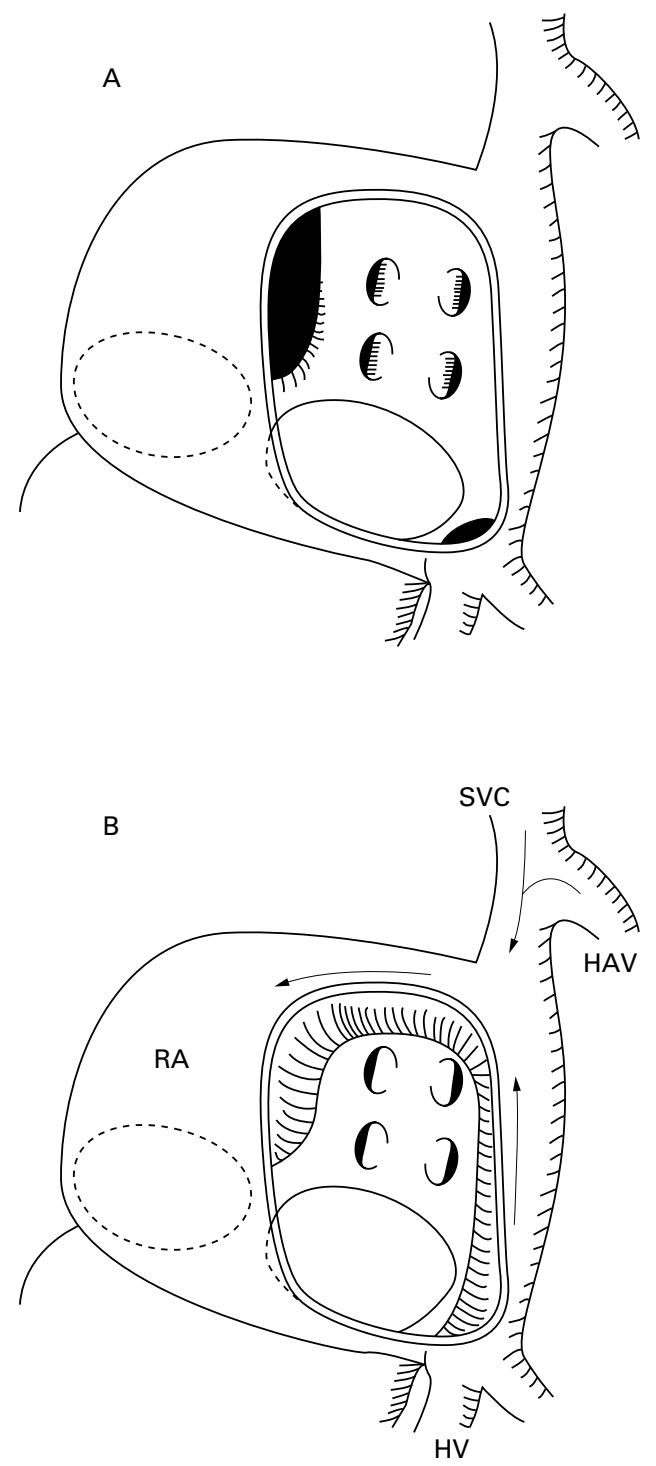

Figure 1 (A) Preoperative atrial and caval anatomy. (B) Operative procedures. HAV, hemiazygous vein; $H V$, hepatic vein; $R A$, right sided atrium; $S V C$, superior vena cava.

SURGERY

Biventricular corrective repair was performed when she was 5 years old. The left ventricle and aorta were connected with an internal conduit 


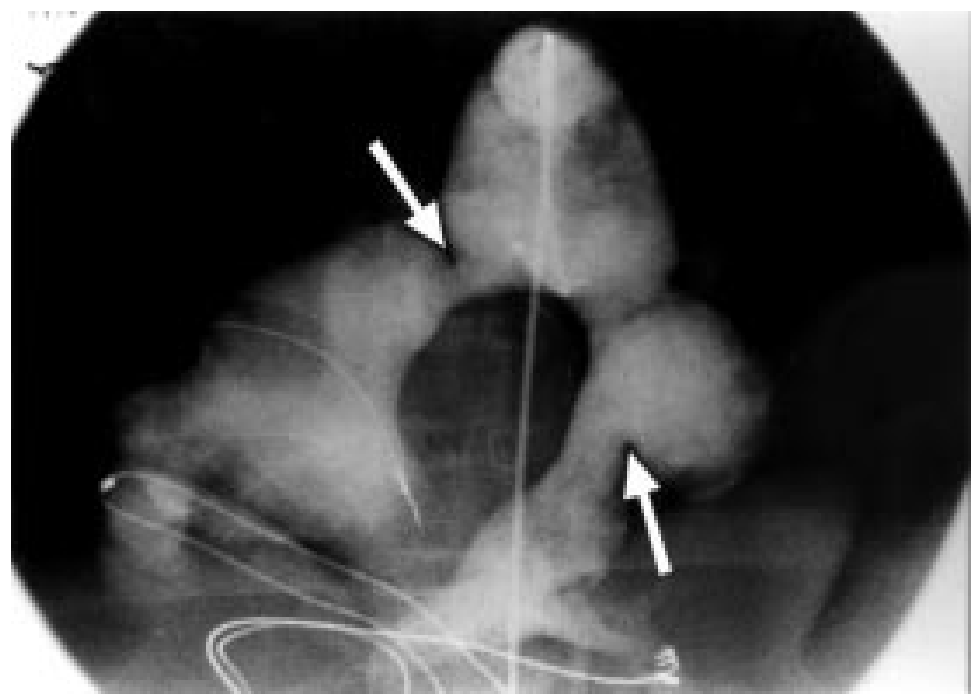

Figure 2 Angiogram before stent implantation. Arrows indicate baffle stenoses.

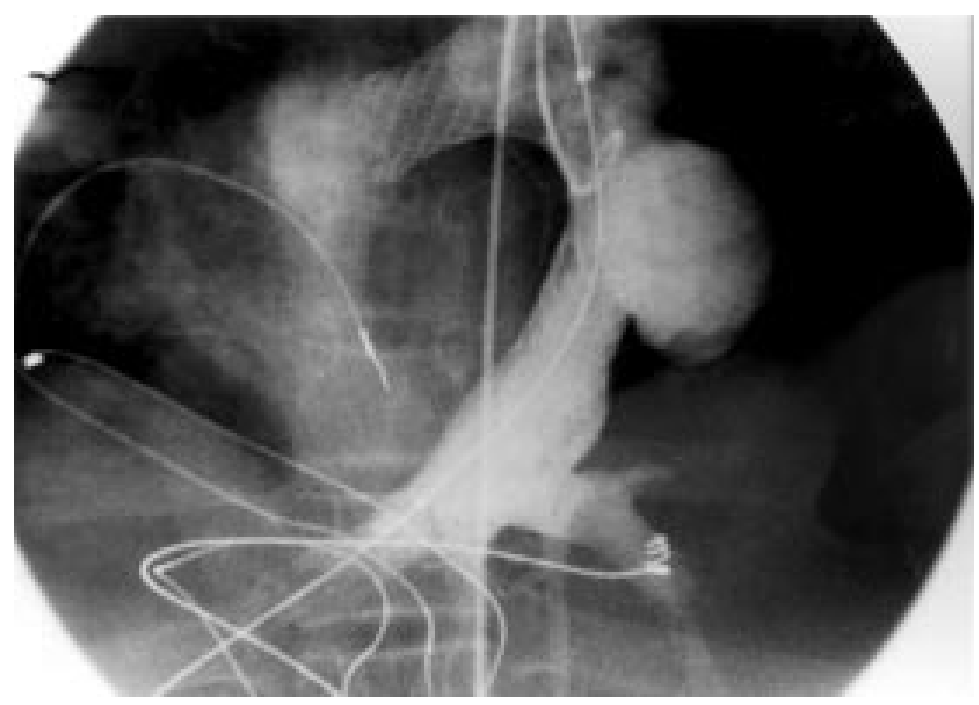

Figure 3 Angiogram after stent implantation.

through the subaortic ventricular septal defect and a right ventricular outflow tract was reconstructed with a transannular patch bearing a monocusp. Intra-atrial rerouting of systemic and pulmonary venous returns was performed with a Dacron patch. The route from hepatic vein to the orifice of the left SVC was placed at the lateral side of the left sided atrium, and this route was connected to the right sided atrium through the atrial septal defect (fig 1). Postoperative course was uneventful and a cardiac pacemaker was implanted because of complete atrioventricular block one month later. She was discharged in a favourable condition.

POSTOPERATIVE COURSE

The patient had been very well during follow up but eight years after surgery she had pronounced abdominal distension with massive ascites. Cardiac catheterisation showed systemic venous baffle stenosis between the hepatic vein and SVC, and between the SVC and right sided atrium. Angiography showed that the width of the channel between the hepatic vein and SVC was $11 \mathrm{~mm}$ and that between the SVC and right sided atrium was $7 \mathrm{~mm}$ (fig 2). Pressure measurement showed $5 \mathrm{~mm} \mathrm{Hg}$ of pressure gradient between the SVC and right sided atrium and no pressure gradient between the hepatic vein and SVC.

BALLOON DILATATION AND STENT PLACEMENT Balloon dilatation was initially attempted with a $15 \mathrm{~mm}$ balloon catheter (Meditech, Mansfield, Massachusetts, USA). The "waist" of the balloon in the stenotic lesion disappeared during inflation but elastic recoil of the stenotic lesion soon occurred and the procedure was unsuccessful.

Seven weeks later, two Palmaz stents of $30 \mathrm{~mm}$ length (Johnson and Johnson Interventional Systems, Sommerville, New Jersey, USA) were placed into the stenotic lesions using a delivery catheter with a $15 \mathrm{~mm}$ diameter balloon inserted from the left internal jugular vein. The first stent was implanted at the stenosis between the SVC and right atrium and the second at that between the hepatic vein and SVC. Angiography confirmed widely open channels (fig 3) and the pressure gradient between the SVC and right atrium disappeared.

FOLLOW UP

The ascites decreased and abdominal distension disappeared soon after stent implantation. The patient has been well since then and echocardiography 15 months after stent implantation showed no recurrence of stenosis.

\section{Discussion}

Stenosis of systemic venous baffles was one of the frequent and serious complications after Mustard operation for transposition of the great arteries. The operative risk for surgical relief was not small because systemic ventricular dysfunction and atrioventricular valve regurgitation were often found in patients who had a right ventricle as a systemic ventricle. ${ }^{3}$ In our patient, the systemic ventricle was the left ventricle, but straddling of the mitral valve had resulted in mild mitral regurgitation and moderate reduction of left ventricular function. Moreover, the stenotic lesions were behind the ventricles and great arteries and it was difficult to reach these lesions without cardiopulmonary bypass or cardioplegic arrest. We, therefore, decided to dilate the stenotic lesions using balloon dilatation.

There are several reports that mention efficacy of balloon dilatation for stenotic venous vessels, ${ }^{5}$ although the mechanism of dilatation was uncertain. ${ }^{6}$ In our patient, the walls of the stenotic segments consisted of atrial tissue and Dacron patch, and balloon dilatation was unsuccessful because elastic recoil occurred immediately after dilatation. In these stenotic lesions, balloon expandable stents are useful to provide complete relief from both haemodynamic and angiographic obstructions.

Stenosis or occlusion by intimal overgrowth or thrombosis might interfere with patency of the stent. Twelve of 14 implanted stents were patent from 56 to 278 days after implantation in an animal model examined by Mullins et al. ${ }^{7}$ 
These stents were covered with neointima and no thrombus was found. Good intermediate term patency of the stents after implantation was also reported in patients in both pulmonary arteries and systemic veins. ${ }^{7}$

Another possible mechanism of restenosis after stent implantation is relative stenosis of the blood vessels after growth of the body. This Palmaz stent is effective even if the stent is not fully expanded and we think a larger sized stent should be implanted into small paediatric patients. Redilatation of the stent could fully expand it and the restenosis could be relieved. ${ }^{8}$

In conclusion, we successfully implanted two Palmaz stents for relief of venous baffle stenoses after atrial rerouting surgery. These lesions could not be relieved by balloon dilatation alone.

We sincerely thank Charles E Mullins (Texas Children's Hospital) for his instructive and technical assistance.
1 Chatelain P, Meier B, Friedli B. Stenting of superior vena cava and inferior vena cava for symptomatic narrowing after repeated atrial surgery for D-transposition of the great vessels. Br Heart F 1991;66:466-8.

2 O'Laughlin MP, Slack MC, Grifka RG, et al. Implantation and intermediate-term follow-up of stents in congenital heart disease. Circulation 1993;88:605-14.

3 Abdulhamed JM, Yousef SA, Khan MAA, et al. Balloon dilation of complete obstruction of the superior vena cava after Mustard operation for transposition of great arteries. Br Heart $\mathcal{f} 1994 ; 72: 482-5$.

4 Ward CJB, Mullins CE, Nihill MR, et al. Use of intravascular stents in systemic venous and systemic venous baffle obstructions, short-term follow-up results. Circulation 1995;91:2948-54.

5 Wisselink W, Money SR, Becker MO, et al. Comparison of operative reconstruction and percutaneous balloon dilation for central venous obstruction. Am F Surg 1993;166:200-5.

6 Lock JE, Bass JL, Castaneda-Zuniga W, et al. Dilation angioplasty of congenital or operative narrowings of venous channels. Circulation 1984;70:457-64.

7 Mullins CE, O'Laughlin MP, Vick W, et al. Implantation of balloon-expandable intravascular grafts by catheterization in pulmonary arteries and systemic veins. Circulation 1988; 77:188-99.

8 Trerotola SO, Lund GB, Samphilipo MA, et al. Palmaz stent in the treatment of central venous stenosis: safety and efficacy of redilation. Radiology 1994;190:379-85. 